\section{"Optische Biopsie" erspart unnötige Exzisionen}

Die konfokale Lasermikroskopie (CLSM) erlaubt eine nicht invasive, hochauflösende Visualisierung von Details der Hautzellen in vivo und bietet als "optische Biopsie“ ein rasches Ergebnis als Entscheidungsgrundlage für oder wider eine Therapie.

Der Schwerpunkt bei dieser Methode liegt auf melanozytären Läsionen. Im Vergleich zur Dermatoskopie verbessert die CLSM die Spezifität ohne Unterschied in der Sensitivität, so Dr. med. Martina Ulrich, Dermatologie am Regierungsviertel, CMB Collegium Medicum Berlin. Damit lassen sich unnötige Exzisionen gutartiger Hautveränderungen vermeiden. Bezüglich des Basalzellkarzinoms erreicht die CLSM eine Sensitivität von $100 \%$ und eine Spezifität von über $88 \%$ (Guitera P et al. J Invest Dermatol 2012;132:2386-94).

\section{Verbesserung der diagnostischen Genauigkeit}

Die S3-Leitlinie „Prävention von Hautkrebs" beurteilt die CLSM als hochauflösende Methode bei der Beurteilung pigmentierter und nicht pigmentierter Läsionen der Haut. Nach entsprechendem Training kann die CLSM die diagnostische Genauigkeit dieser Läsionen verbessern, so der Tenor der Leitlinie.

Anwender erhalten in einem kostenlosen, modular aufgebauten Trainingsprogramm Einführungstrainings, Module für das Training in Eigenregie, OnlineSchulungen, ergänzende Studienmaterialien und Lehrbücher. Die bisher erschienenen 537 Abstracts zur CLSM wurden in einem Online-Booklet unter www.vivascopepub.com zusammengestellt.
Die Vorteile der CLSM liegen in den vielfältigen Anwendungsmöglichkeiten in der täglichen Praxis, betonte Ulrich. Dazu gehören die Primärdiagnostik von melanozytären und nicht melanozytären Hauttumoren, das therapeutische Monitoring, die Anwendung in klinischen Studien und die Bestimmung von Tumorgrenzen. Aufgrund der hohen Sensitivität und Spezifität der Methode und sofortiger Diagnosestellung bleiben dem Patienten unnötige Biopsien erspart, so Ulrich.

Als Ausblick in die nähere Zukunft nannte Ulrich u.a. die Ferndiagnose durch Konfokal-Experten mittels Telemedizin und Teledermatologie $\left(\right.$ VivaNet $^{\circledR}$ ) und den Ex-vivo-Einsatz zur mikrographisch kontrollierten Chirurgie.

Wolfgang Zimmermann

Praktisches Fortbildungsprogramm "Nicht-invasive Diagnostik von Hautkrebs" im Rahmen des 25. Deutschen Hautkrebskongresses der Arbeitsgemeinschaft Dermatologische Onkologie, München, 12.9.2015; Veranstalter: MAVIG, VivaScope Systems, Michelson Diagnostics und SciBase

\title{
UIP-Forschungsstipendium und Travel Award 2015
}

Im Rahmen des diesjährigen UIP World Meetings vom 27.-29. August in Seoul, Südkorea, gaben die Union Internationale de Phlébologie (UIP) und Kreussler Pharma den Gewinner des Kreussler Young Scientists' Sclerotherapy Awards 2015 bekannt. Das mit 10.000 Euro dotierte Forschungsstipendium unterstützt junge Wissenschaftler bei der Durchführung einer klinischen, präklinischen oder experimentellen Studie zum Thema Sklerotherapie von Varizen.

Der diesjährige Gewinner, Dr. Roshan Bootun aus London, Großbritannien, plant eine randomisierte, kontrollierte klinische Studie zur Wirksamkeit der Kompressionstherapie nach der endovenösen Behandlung von Varizen mit der Schaum-Sklerotherapie mit Polidocanol (in Deutschland unter dem Namen Aethoxysklerol $^{\circledast}$ im Handel). Obwohl viele Leitlinien die Anwendung der Kom- pression für bestimmte Indikationen empfehlen, verbleibt eine gewisse Unsicherheit über den Nutzen von Kompressionsstrümpfen nach der Behandlung von Varizen mit der Sklerotherapie. Die Hauptziele der Studie von Dr. Bootun sind, die Schmerzen der Patienten während der ersten 10 Tage nach der Sklerotherapie mittels der validierten visuellen Analogskala (VAS) zu untersuchen und die zwei Behandlungsgruppen mittels eines „Quality of Life“ Scores zu vergleichen. Die Ergebnisse der Studie werden 2018 beim nächsten UIP Meeting präsentiert und sollten wichtige neue Erkenntnisse über den positiven Effekt der Kompression nach der Behandlung von Varizen mit der Schaum-Sklerotherapie ergeben. „Der Kreussler Young Scientists' Sclerotherapy Award stellt für junge Phlebologen eine sehr gute Möglichkeit dar, ihre kreativen Ideen zu phle-

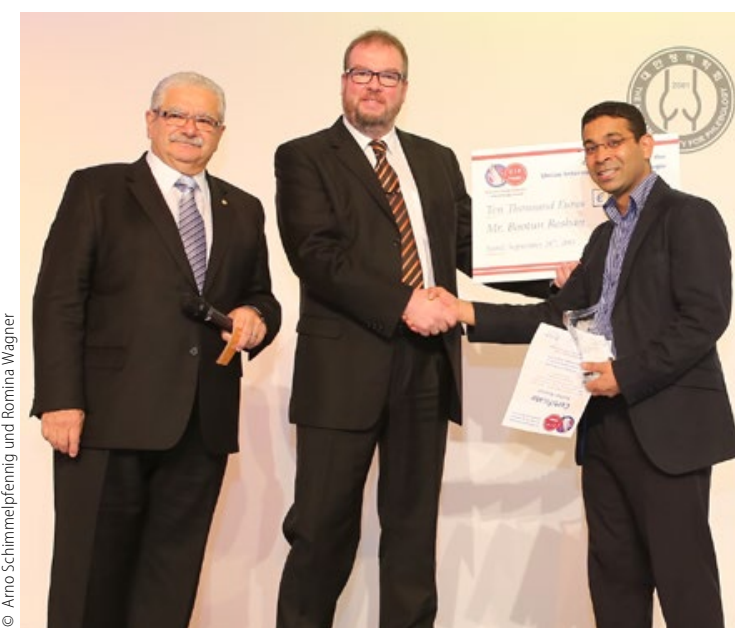

UIP-Award-Gewinner Dr. Roshan Bootun (rechts) bei der Verleihung des Awards auf dem UIP 2015

bologischen Fragestellungen in der klinisch-medizinischen Forschung in die Tat umzusetzen“, so Prof. Dr. med. Eberhard Rabe, Past-Präsident der UIP, der den diesjährigen Gewinner zusammen mit einem Expertenkomitee auswählte.

Nach Informationen von Kreussler Pharma 